\title{
Circulating 25-hydroxyvitamin D levels in relation to blood pressure parameters and hypertension in the Shanghai Women's and Men's Health Studies
}

\author{
Tsogzolmaa Dorjgochoo ${ }^{1}$, Xiao Ou Shu ${ }^{1 *}$, Yong-Bing Xiang ${ }^{2}$, Gong Yang ${ }^{1}$, Qiuyin Cai ${ }^{1}$, Honglan Li $^{2}$, \\ Bu-Tian $\mathrm{Ji}^{3}$, Hui Cai ${ }^{1}$, Yu-Tang $\mathrm{GaO}^{2}$ and Wei Zheng ${ }^{1}$ \\ ${ }^{1}$ Division of Epidemiology, Department of Medicine and Vanderbilt Epidemiology Center, Vanderbilt University School of \\ Medicine, 2525 West End Avenue, Suite 600, IMPH, Nashville, TN 37203, USA \\ ${ }^{2}$ Department of Epidemiology, Shanghai Cancer Institute, Shanghai 200032, People's Republic of China \\ ${ }^{3}$ Division of Cancer Epidemiology and Genetics, National Cancer Institute, Bethesda, MD 20892, USA
}

(Submitted 10 January 2011 - Final revision received 14 September 2011 - Accepted 25 September 2011 - First published online 27 February 2012)

\section{Abstract}

Little is known about the association of circulating 25-hydroxyvitamin D (25(OH)D) and blood pressure (BP) parameters, including systolic and diastolic BP, pulse pressure (PP), mean arterial pressure (MAP) and hypertension in non-Western populations that have not yet been exposed to foods fortified with vitamins and seldom use vitamin D supplements. A cross-sectional analysis of plasma 25(OH)D levels in association with BP measures was performed for 1460 participants ( 1055 women and 405 men, aged 40-74 years) of two large cohort studies in Shanghai. Multivariable linear and logistic regressions were conducted. Overall, the prevalence of vitamin D deficiency was $55.8 \%$ using National Health and Nutrition Examination Survey, USA criteria and $29 \cdot 9 \%$ using WHO criteria. The median plasma $25(\mathrm{OH}) \mathrm{D}$ level in the population was $38.0 \mathrm{nmol} / 1$ for men and $33.6 \mathrm{nmol} / 1$ for women $(P<0 \cdot 01)$ among participants who were not on antihypertensive drugs. Among men, BP parameters (systolic BP, diastolic BP and MAP) were significantly and inversely associated with higher quintiles of $25(\mathrm{OH}) \mathrm{D}$ compared with the lowest quintile $\left(P_{\text {trend }}<0.05\right.$ for all). Vitamin D non-deficient status (WHO criteria) was inversely associated with hypertension $\left(\mathrm{OR}_{\text {adjusted }}=0 \cdot 29 ; 95 \% \mathrm{CI} 0 \cdot 10,0 \cdot 82\right)$. An inverse association was also found between hypertension and the highest quintile of $25(\mathrm{OH}) \mathrm{D}\left(\mathrm{OR}_{\text {adjusted }}=0.16 ; 95 \% \mathrm{CI} 0.04,0.65\right.$ for $\left.\geq 50.6 \mathrm{nmol} / \mathrm{l} ; P_{\text {trend }}=0.02\right)$. Among women, no significant associations were found for BP parameters and hypertension. The present study shows that vitamin D deficiency is common among adults in urban China. Circulating 25(OH)D levels were inversely related to the levels of individual BP parameters and hypertension among middle-aged and elderly men but not among women. More research is needed to investigate the potential sex differential associations.

Key words: Blood pressure parameters: Hypertension: 25-Hydroxyvitamin D: Sex differences: China

Experimental data indicate that vitamin $\mathrm{D}$ is involved in various physiological functions in humans, including cellular inflammation, oestrogen biosynthesis, regulating $\mathrm{Ca}$ homeostasis and the renin-angiotensin system, and vascular muscle function $^{(1,2)}$. Vitamin D deficiency has been associated with increased risk of numerous ageing-related chronic diseases, particularly hypertension, CVD and osteoporosis ${ }^{(3,4)}$. However, the mechanisms by which vitamin $\mathrm{D}$ influences these medical conditions remain unclear. Sunlight exposure is a major determinant of vitamin D status, because vitamin $\mathrm{D}$ is naturally present in very few foods ${ }^{(3)}$. The circulating 25-hydroxyvitamin D $(25(\mathrm{OH}) \mathrm{D})$ concentration is the most reliable biomarker of vitamin D status, but differs in humans by geographic location, status of food fortification, vitamin supplementation and the varying intensity of UV light to which they are exposed ${ }^{(3,5)}$. Moreover, skin pigmentation, age, sex, lifestyle factors, diet, vitamin D supplementation and genetic factors influence vitamin D status ${ }^{(6-8)}$.

Some epidemiological studies ${ }^{(9-11)}$, including recent systematic review and a meta-analysis of three cohorts ${ }^{(12)}$ conducted in Western countries, have shown that lower levels of $25(\mathrm{OH}) \mathrm{D}$ may increase the risk of high blood pressure (BP) or hypertension; however, other studies ${ }^{(13)}$ including randomised clinical trials ${ }^{(14,15)}$ found no such association or found a positive association between vitamin $\mathrm{D}$ level and $\mathrm{BP}$ that depends on seasonality ${ }^{(15,16)}$ or genetic factors ${ }^{(8)}$. Studies

\footnotetext{
Abbreviations: 1,25(OH)2D, 1,25 dihydroxy vitamin D; 25(OH)D, 25-hydroxyvitamin D; BP, blood pressure; MAP, mean arterial pressure; MET, metabolic equivalents; NHANES, National Health and Nutrition Examination Survey; PP, pulse pressure; PTH, parathyroid hormone; SMHS, Shanghai Men's Health Study; SWHS, Shanghai Women's Health Study; VDPP, Vitamin D Pooling Project.
} 
that comprehensively examine the association of vitamin $\mathrm{D}$ status with BP parameters (systolic BP, diastolic BP, pulse pressure (PP) and mean arterial pressure (MAP)) and clinical categories of BP (normal, pre-hypertension and hypertension) in both men and women are scarce. In addition, to our knowledge, no such study has been conducted in the Chinese population. Unlike Western populations, the Chinese population has not yet been exposed to foods fortified with vitamin $\mathrm{D}$ and seldom uses vitamin D supplements or sunscreen products, all of which may affect vitamin D status ${ }^{(5)}$. Thus, it remains unknown whether findings on the association between circulating vitamin D levels and BP found in Western populations can be directly generalised to Chinese populations.

This report describes a comprehensive evaluation of the effect of circulating 25(OH)D levels on individual $\mathrm{BP}$ parameters (systolic BP, diastolic BP, PP and MAP) and on the prevalence of pre-hypertension and hypertension using data from two longitudinal studies of over 135000 middleaged and elderly Chinese men and women. In recent decades, China has experienced a rapid increase in cardiovascular morbidity and mortality, presumably driven by elevated $\mathrm{BP}$ or hypertension ${ }^{(17,18)}$. The findings of the present study could help with the development of effective lifestyle and dietary interventions to decrease the risk of CVD morbidity and mortality in both men and women.

\section{Subjects and methods}

\section{Participants}

The participants of the present cross-sectional study came from two large, population-based, prospective cohort studies conducted in Shanghai, China. The Shanghai Women's Health Study (SWHS) recruited 74941 women aged 40-70 years between 1996 and 2000 (response rate: 92.7\%), while the Shanghai Men's Health Study (SMHS) recruited 61491 men aged 40-74 years between 2002 and 2006 (response rate: $74 \cdot 1 \%$ ). The SWHS and SMHS followed virtually identical recruitment and follow-up protocols and used similar survey instruments; detailed study protocols have been published elsewhere $^{(19,20)}$. Briefly, permanent residents of seven (for the SWHS) and eight (for the SMHS) urban districts of Shanghai who met the age eligibility criteria were recruited into these studies through in-home visits by trained interviewers. Participants were followed-up via biennial questionnaires that gathered information on diet, lifestyle and medical events. At baseline, 56830 women and 46119 men contributed blood samples that were processed and stored at $-80^{\circ} \mathrm{C}$ within $6 \mathrm{~h}$ until laboratory analysis could be performed. This study was conducted according to the guidelines laid down in the Declaration of Helsinki and all procedures involving human subjects/patients were approved by the Institutional Review Boards of Vanderbilt University and the Shanghai Cancer Institute. Written informed consent was obtained from all participants before interview. The present analysis includes 1055 SWHS participants and 405 SMHS participants, who were included in the Cohort Consortium Vitamin D
Pooling Project (VDPP) of Rarer Cancers (endometrial, oesophageal, gastric, kidney, non-Hodgkin lymphoma, ovarian and pancreatic cancers) as controls. The VDPP included ten prospective cohort studies in the USA, Finland and China (i.e. the SMHS and the SWHS), with stored blood samples and was aimed to investigate the association between circulating $25(\mathrm{OH}) \mathrm{D}$ levels and rarer cancers using a nested casecontrol study approach ${ }^{(21)}$.

\section{Blood pressure measurement and ascertainment of blood pressure categories}

BP was measured for women at the first follow-up survey (2000-02) and for men at the baseline (2002-06) survey. BP measurements (systolic and diastolic BP) were taken uniformly according to standard protocols. Participants were asked to sit and relax. After at least a $5 \mathrm{~min}$ rest, heart rate was measured by manual pulse palpation over a period of $30 \mathrm{~s}$, and BP was measured using the auscultatory method with a conventional mercury sphygmomanometer. Two BP measurements were taken at $30 \mathrm{~s}$ intervals, and an average of these two $\mathrm{BP}$ readings (in $\mathrm{mmHg}$ ) was used in this analysis. The aneroid devices were calibrated every 6 months by staff from the Shanghai Municipal Bureau of Quality and Technical Supervision during the recruitment period.

History of hypertension was defined as having physiciandiagnosed hypertension and/or using antihypertensive medication based on self-reported information before BP measurement and blood sampling. Participants were categorised hierarchically into: normal BP (systolic BP $<120 \mathrm{mmHg}$ and diastolic $\mathrm{BP}<80 \mathrm{mmHg}$ ), pre-hypertension (not normal BP and 120-139 and/or 80-89 $\mathrm{mmHg}$ ) and hypertension (systolic $\mathrm{BP} \geq 140 \mathrm{mmHg}$ or diastolic $\mathrm{BP} \geq 90 \mathrm{mmHg}$ ) according to the criteria recommended by the Joint National Committee 7 on Prevention, Detection, Evaluation and Treatment of High Blood Pressure ${ }^{(22)}$. The Joint National Committee 7 criteria for $\mathrm{BP}$ categories is the most widely used classification for assessing high BP in China. Participants on antihypertensive medication were classified into the hypertension group. PP was defined as the difference between systolic BP and diastolic BP. MAP was calculated as diastolic BP plus one-third PP.

\section{Measurement of plasma 25-hydroxyvitamin D}

This study included samples that were selected specifically for $25(\mathrm{OH}) \mathrm{D}$ measurement as part of the $\mathrm{VDPP}^{(18)}$. The measurement of $25(\mathrm{OH}) \mathrm{D}$ in $125 \mu \mathrm{l}$ samples of plasma was performed by Heartland Assays, Inc. (Ames, IA, USA), which is the central laboratory used by the VDPP, using the 'LIAISON ${ }^{\circledR}$ 25-OH Vitamin D TOTAL' chemiluminescent immunoassay $^{(21)}$. Blinded quality control samples were included within each sample batch to assess assay reproducibility. Both the median inter- and intra-assay CV for $25(\mathrm{OH}) \mathrm{D}$ measurements were 11.0 and $13.6 \%$, respectively, as described in detail elsewhere ${ }^{(21)}$. Circulating $25(\mathrm{OH}) \mathrm{D}$ is extremely stable and its measurements are not affected by multiple freeze-thaw cycles. 


\section{Ascertainment of covariates}

The exposure assessments for both cohorts were essentially identical, except for reproductive and medical information, which was sex-specific. At baseline, information about sociodemographic characteristics, reproductive history, medical history, dietary and lifestyle habits (smoking habits and alcohol and tea consumption), weight history, physical activity, family history of chronic disease and occupational history was collected, and weight, height, waist circumference and hip circumference measurements were taken according to a standard protocol. Usual dietary intake was assessed at the in-person interview using a validated FFQ and intake of nutrients $(\mathrm{g} / \mathrm{d}$ or $\mathrm{mg} / \mathrm{d})$ and total energy $(\mathrm{kJ} / \mathrm{d})$ was estimated using the Chinese Food Composition Tables ${ }^{(23,24)}$.

\section{Statistical analysis}

The level of plasma 25(OH)D (independent variable) was not normally distributed (slightly right-skewed), whereas BP (dependent variable) was normally distributed. Log-transformation was conducted to approach normal distribution for plasma 25(OH)D. Demographic characteristics and established risk factors for hypertension in relation to plasma 25(OH)D levels (median, 25th and 75th percentiles) were evaluated for men and women by using the Wilcoxon ranksum test for categorical variables and Spearman's rank correlation test (correlation coefficient) for continuous variables. Plasma 25(OH)D levels were evaluated in quintile distributions, with the lowest quintile defined as the reference category. In addition, 25(OH)D level was categorised using the following clinically relevant common cut-off points: deficient $(<37.5 \mathrm{nmol} / \mathrm{l})$, insufficient $(37.5-74.9 \mathrm{nmol} / \mathrm{l})$ and sufficient $(\geq 75.0 \mathrm{nmol} / \mathrm{l})$, according to the Third National Health and Nutrition Examination Survey (NHANES-III, USA) ${ }^{(11,25)}$. We further examined the deficient $(<27.5 \mathrm{nmol} / \mathrm{l})$ and nondeficient $(\geq 27.5 \mathrm{nmol} / \mathrm{l})$ categories set out by the World Health Organization ${ }^{(26)}$. Vitamin D deficiency status was used as the reference group throughout the analyses. We also examined the association of BP categories with $25(\mathrm{OH}) \mathrm{D}$ level as a continuous variable (overall in log-transformed $25(\mathrm{OH}) \mathrm{D}$ and each five unit increase). BP (systolic BP, diastolic BP, PP and MAP) differences in $25(\mathrm{OH}) \mathrm{D}$ levels were explored to estimate the mean difference (non-standardised $\beta$ coefficient) and its 95\% CI in multivariate linear regression models. Participants on antihypertensive medication were excluded from the analysis of BP measurements. We also estimated standardised regression coefficients of $\mathrm{BP}$ measures with $25(\mathrm{OH}) \mathrm{D}$ and found similar association patterns. We chose to present the results of non-standardised regression analysis because they are clinically more relevant than the results generated from standardised regression analysis.

Models were adjusted for age, BMI, family history of hypertension, education, occupation, alcohol consumption, smoking, physical activity (both self-reported and expressed in metabolic equivalents (MET)), intakes of total energy, eggs and vitamin $\mathrm{D}$, and season of blood collection. These covariates were chosen based on their associations with circulating
25(OH)D and as established risk factors for hypertension. Dietary intakes of $\mathrm{Ca}$ and $\mathrm{Na}$ were considered to be potential confounders, but were not included in the final models as they did not appreciably alter the mean BP differences. Highly correlated variables, such as systolic BP, diastolic BP, PP, MAP, age and menopausal status, were not included in the same model. We performed sex-specific analyses and analyses of men and women combined with additional adjustment for sex.

We evaluated the associations of $25(\mathrm{OH}) \mathrm{D}$ levels with BP categories (among normotensive, pre-hypertensive and hypertensive subjects) using polytomous logistic regression to estimate OR and 95\% CI stratified by sex and for all participants combined. Models were adjusted for the aforementioned variables. Dose-response trends were analysed by treating ordinal scores of the categorical variables as a continuous variable in the model. Tests for interaction were performed by introducing a multiplicative interaction term into the main effect models. All statistical tests were based on two-tailed probability and a significance level set at $\alpha<0.05$ and performed using SAS statistical software, version 9.2 (SAS Institute, Cary, NC, USA).

\section{Results}

The median age of the population was 61 years, women were younger than men (59v. 66 years; $P<0.001$ ). Overall, the median 25(OH)D level was $34.7 \mathrm{nmol} / 1$, which differed considerably between men and women (38.0 and 33.6, respectively, $P<0.001$ ) who were not on antihypertensive medication (data not shown). The circulating 25(OH)D levels (median, 25th and 75th percentile) in relation to each category of the selected demographic and established risk factors for hypertension and correlation coefficients of $25(\mathrm{OH}) \mathrm{D}$ levels with age, physical activity (MET), BMI and dietary intakes in all participants by sex are shown in Table 1. Lower 25(OH)D levels were found among women with low levels of education $(P=0.07)$ or less-skilled occupations $(P<0 \cdot 01)$, compared with women with higher levels of education or occupation. Both men and women who ever smoked were more likely to have lower $25(\mathrm{OH}) \mathrm{D}$ levels. Interestingly, men who consumed alcohol regularly had higher $25(\mathrm{OH}) \mathrm{D}$ levels. 25(OH)D levels differed by the season in which the blood samples had been collected. In general, high levels of plasma 25(OH)D were found during the period from June to November, while lower levels were found from December to May in both men and women. Physical activity and intake of eggs or dietary vitamin $\mathrm{D}$ were positively correlated with $25(\mathrm{OH}) \mathrm{D}$ level among women. There was a positive correlation between age and self-reported regular physical activity and 25(OH)D levels among men.

Analyses restricted to non-users of hypertensive drugs showed that the association between plasma $25(\mathrm{OH}) \mathrm{D}$ and BP measurement varied by sex (Table 2). Among men, all BP parameters were lower among those with higher quintiles of $25(\mathrm{OH}) \mathrm{D}$ ( $P_{\text {trend }}<0.05$ for all, except for PP). Similar results were observed when $25(\mathrm{OH}) \mathrm{D}$ level was analysed as a continuous variable. Among women, only PP tended to be lower among those with higher quintiles of $25(\mathrm{OH}) \mathrm{D}$ level 
Table 1. Circulating 25-hydroxyvitamin $\mathrm{D}(25(\mathrm{OH}) \mathrm{D})$ levels $(\mathrm{nmol} / \mathrm{l})$ in relation to selected demographic and established risk factors for hypertension among men and women in the Shanghai Women's Health Study and Shanghai Men's Health Study, China (Median values and 25th (P25)-75th percentiles (P75))

\begin{tabular}{|c|c|c|c|c|c|c|}
\hline \multirow[b]{2}{*}{ Risk factors } & \multicolumn{3}{|c|}{ Men $(n$ 405) } & \multicolumn{3}{|c|}{ Women ( $n$ 1055) } \\
\hline & Median & $\mathrm{P} 25-\mathrm{P} 75$ of $25(\mathrm{OH}) \mathrm{D}$ & $P^{*}$ & Median & $\mathrm{P} 25-\mathrm{P} 75$ of $25(\mathrm{OH}) \mathrm{D}$ & $P^{*}$ \\
\hline \multicolumn{7}{|l|}{ Education } \\
\hline$\leq$ Primary & 40.4 & $31 \cdot 8-53.9$ & & $32 \cdot 7$ & $23.5-44.5$ & \\
\hline Middle school & 41.9 & $29 \cdot 0-55 \cdot 4$ & & $34 \cdot 2$ & $24 \cdot 0-43.5$ & \\
\hline High school & $37 \cdot 3$ & $27 \cdot 5-49 \cdot 8$ & & 34.5 & $25 \cdot 3-48 \cdot 1$ & \\
\hline$\geq$ College & $37 \cdot 3$ & $29 \cdot 0-55 \cdot 2$ & 0.25 & $36 \cdot 2$ & $27 \cdot 3-48 \cdot 4$ & 0.07 \\
\hline \multicolumn{7}{|l|}{ Occupation } \\
\hline Professional & 39.1 & $30.4-54.9$ & & 34.8 & $26 \cdot 3-49 \cdot 0$ & \\
\hline Clerical & $36 \cdot 5$ & $26 \cdot 8-55 \cdot 4$ & & $36 \cdot 9$ & $25 \cdot 9-46 \cdot 8$ & \\
\hline Manual labourers & $40 \cdot 0$ & $29 \cdot 6-50 \cdot 8$ & 0.77 & $32 \cdot 2$ & $23 \cdot 5-42 \cdot 4$ & $<0.01$ \\
\hline \multicolumn{7}{|l|}{ Smoking status } \\
\hline Never & 41.4 & $31 \cdot 7-54.5$ & & $34 \cdot 2$ & $25 \cdot 0-45 \cdot 6$ & \\
\hline Ever & 37.5 & $27 \cdot 2-50 \cdot 9$ & 0.05 & $28 \cdot 8$ & $20 \cdot 6-39 \cdot 0$ & $<0.01$ \\
\hline \multicolumn{7}{|l|}{ Regular alcohol consumption } \\
\hline Never & $38 \cdot 3$ & $29 \cdot 0-51 \cdot 0$ & & 33.8 & $24 \cdot 7-45 \cdot 3$ & \\
\hline Ever & $42 \cdot 4$ & $29 \cdot 0-57.5$ & 0.26 & $37 \cdot 6$ & $26 \cdot 8-45 \cdot 1$ & 0.63 \\
\hline \multicolumn{7}{|l|}{ Regular exercise } \\
\hline Never & $36 \cdot 9$ & $28 \cdot 0-50 \cdot 2$ & & 33.7 & $24 \cdot 2-45 \cdot 9$ & \\
\hline Ever & $40 \cdot 7$ & $29 \cdot 6-57 \cdot 1$ & 0.06 & $34 \cdot 1$ & $25 \cdot 2-44 \cdot 7$ & 0.76 \\
\hline \multicolumn{7}{|l|}{ Family history of hypertension } \\
\hline Never & $41 \cdot 8$ & $30 \cdot 7-55 \cdot 4$ & & 33.4 & $24 \cdot 1-44 \cdot 5$ & \\
\hline Ever & $36 \cdot 9$ & $27 \cdot 5-49 \cdot 9$ & & $34 \cdot 3$ & $25 \cdot 8-45 \cdot 9$ & \\
\hline Unknown & $35 \cdot 9$ & $27 \cdot 9-50 \cdot 7$ & 0.12 & $32 \cdot 4$ & $22 \cdot 9-45 \cdot 9$ & 0.47 \\
\hline \multicolumn{7}{|l|}{ Season of blood draw } \\
\hline Winter-spring (December-May) & 33.0 & $24 \cdot 0-43 \cdot 6$ & & $29 \cdot 6$ & $22 \cdot 0-38 \cdot 1$ & \\
\hline \multirow[t]{2}{*}{ Summer-autumn (June-November) } & $45 \cdot 3$ & $34 \cdot 7-60 \cdot 1$ & $<0.01$ & $40 \cdot 3$ & $29 \cdot 7-51 \cdot 8$ & $<0.01$ \\
\hline & \multicolumn{2}{|c|}{ Correlation coefficient } & $P \dagger$ & \multicolumn{2}{|c|}{ Correlation coefficient } & $P$ \\
\hline Age (years) & & 0.12 & 0.02 & & -0.03 & 0.30 \\
\hline Total physical activity (MET h/week) & & 0.06 & 0.21 & & 0.07 & 0.02 \\
\hline BMI $\left(\mathrm{kg} / \mathrm{m}^{2}\right)$ & & -0.05 & 0.31 & & -0.04 & 0.22 \\
\hline Total energy (kJ/d) & & 0.06 & 0.24 & & 0.02 & 0.62 \\
\hline Total fat $(\mathrm{g} / \mathrm{d})$ & & 0.07 & 0.15 & & 0.04 & 0.15 \\
\hline Eggs $(g / d)$ & & 0.03 & 0.52 & & 0.13 & $<0.01$ \\
\hline Dietary vitamin D intake (lu/d) & & 0.08 & 0.13 & & 0.09 & $<0.01$ \\
\hline $\mathrm{Na}(\mathrm{mg} / \mathrm{d})$ & & 0.03 & 0.50 & & 0.02 & 0.44 \\
\hline $\mathrm{Ca}(\mathrm{mg} / \mathrm{d})$ & & -0.01 & 0.99 & & 0.04 & 0.24 \\
\hline $\mathrm{K}(\mathrm{mg} / \mathrm{d})$ & & 0.01 & 0.89 & & 0.003 & 0.92 \\
\hline
\end{tabular}

MET, metabolic equivalents.

* Wilcoxon rank-sum test for categorical variables.

† Spearman's rank correlation test for continuous variables.

( $\beta=-2 \cdot 19 ; 95 \% \mathrm{CI}-4.96,0.57$ for the highest quintile of 25(OH)D level); however, the results were not significant $\left(P_{\text {trend }}=0 \cdot 16\right)$. A similar non-significant association was observed when $25(\mathrm{OH}) \mathrm{D}$ was analysed as a continuous variable. There was a significant interaction between sex and $25(\mathrm{OH}) \mathrm{D}$ levels (both categorical and continuous variables) on all BP measurements $\left(P_{\text {interaction }}<0.05\right.$ for all, except for $\mathrm{PP}$, data not shown). In the analyses of men and women combined, there were no statistically significant associations between circulating 25(OH)D and systolic BP, diastolic BP, PP or MAP regardless of whether the levels of circulating $25(\mathrm{OH}) \mathrm{D}$ were analysed categorically or continuously.

In our study population, the prevalence of vitamin $\mathrm{D}$ deficiency $(<37.5 \mathrm{nmol} / \mathrm{l})$ was $55.8 \%$, while the prevalence of vitamin D sufficiency was only $3.9 \%(\geq 75.0 \mathrm{nmol} / \mathrm{l})$ using the NHANES-III, USA criteria. When we applied the WHO's criteria for vitamin D status, the rate for vitamin D deficiency $(<27.5 \mathrm{nmol} / \mathrm{l})$ was $29.9 \%$ (data not shown). Prevalence of hypertension $(37 \cdot 5 \%$, systolic $\mathrm{BP} \geq 140 \mathrm{mmHg}$ or diastolic $\mathrm{BP} \geq 90 \mathrm{mmHg}$ and/or use of antihypertensive medication) was common in this population and was higher among men than women $(47.6 \mathrm{v} .33 .6 \% ; P<0.01)$. In the analyses of men and women combined, both pre-hypertension and hypertension tended to be inversely associated with vitamin D sufficiency or with the highest quintile of $25(\mathrm{OH}) \mathrm{D}$ level (Table 3). This inverse association between vitamin D status (i.e. $25(\mathrm{OH}) \mathrm{D}$ level) and pre-hypertension $(\mathrm{OR}=0.57$; $95 \%$ CI $0.26,1.22$ for insufficient status and $0.46 ; 0.14,1.56$ for sufficient status, $P_{\text {trend }}=0 \cdot 11$ ) and hypertension (OR $=0.56 ; 95 \%$ CI $0 \cdot 26,1 \cdot 21$ for insufficient status and $0.42 ; 0.12,1.43$ for sufficient status, $P_{\text {trend }}=0.09$ ) was more pronounced among men than women. A similar association 
Table 2. Mean difference $(\beta)$ and $95 \% \mathrm{Cl}$ individual blood pressure (BP) measurements in relation to circulating 25-hydroxyvitamin $\mathrm{D}(25(\mathrm{OH}) \mathrm{D})$ levels by sex among non-users of antihypertensive drugs in the Shanghai Women's Health Study and Shanghai Men's Health Study*

(Mean difference and $95 \%$ confidence intervals)

\begin{tabular}{|c|c|c|c|c|c|c|c|c|c|c|c|c|}
\hline \multirow[b]{2}{*}{ 25(OH)D (nmol//; quintiles) } & \multicolumn{3}{|c|}{ Systolic BP } & \multicolumn{3}{|c|}{ Diastolic BP } & \multicolumn{3}{|c|}{ PP† } & \multicolumn{3}{|c|}{ MAP $\ddagger$} \\
\hline & $\mathrm{mmHg}$ & $\beta \S$ & $95 \% \mathrm{Cl}$ & $\mathrm{mmHg}$ & $\beta \S$ & $95 \% \mathrm{Cl}$ & $\mathrm{mmHg}$ & $\beta \S$ & $95 \% \mathrm{Cl}$ & $\mathrm{mmHg}$ & $\beta \S$ & $95 \% \mathrm{Cl}$ \\
\hline \multicolumn{13}{|l|}{ Men $(n 260)$} \\
\hline$<23.5$ (lowest) & $132 \cdot 2$ & 0.00 & Referent & $84 \cdot 1$ & 0.00 & Referent & $48 \cdot 1$ & 0.00 & Referent & $100 \cdot 2$ & 0.00 & Referent \\
\hline $23 \cdot 5-31 \cdot 3$ & $127 \cdot 5$ & $-5 \cdot 36$ & $-13.05,2.32$ & 81.9 & -1.52 & $-6.07,3.04$ & $45 \cdot 6$ & -3.85 & $-9.35,1.65$ & $97 \cdot 1$ & $-2 \cdot 80$ & $-7.98,2.38$ \\
\hline $31.4-38.7$ & $128 \cdot 8$ & $-5 \cdot 62$ & $-13 \cdot 39,2 \cdot 14$ & $79 \cdot 7$ & $-5 \cdot 29$ & $-9.89,-0.69$ & $49 \cdot 1$ & -0.33 & $-5 \cdot 89,5 \cdot 22$ & $96 \cdot 0$ & $-5 \cdot 40$ & $-10.63,-0.17$ \\
\hline $38 \cdot 8-50.5$ & $126 \cdot 7$ & $-5 \cdot 65$ & $-13.26,1.95$ & $81 \cdot 6$ & -2.45 & $-6.96,2.06$ & $45 \cdot 0$ & $-3 \cdot 20$ & $-8.65,2.24$ & $96 \cdot 6$ & -3.52 & $-8 \cdot 65,1 \cdot 61$ \\
\hline$\geq 50.6$ (highest) & $124 \cdot 0$ & -9.22 & $-16.90,-1.54$ & $77 \cdot 7$ & $-5 \cdot 23$ & $-9.78,-0.67$ & $46 \cdot 3$ & -3.99 & $-9.49,1.51$ & 93.1 & -6.56 & $-11.73,-1.38$ \\
\hline$P$ trend & & 0.04 & & & 0.04 & & & 0.24 & & & 0.02 & \\
\hline Continuous variable\| & $127 \cdot 2$ & -4.85 & $-8.78,-0.93$ & $80 \cdot 5$ & -2.79 & $-5.05,-0.54$ & $46 \cdot 7$ & -2.06 & $-4.92,0.80$ & $96 \cdot 1$ & -3.48 & $-6.07,-0.89$ \\
\hline Each five unit increase & $127 \cdot 2$ & -0.34 & $-0.78,0.09$ & 80.5 & -0.20 & $-0.45,0.05$ & $46 \cdot 7$ & -0.14 & $-0.46,0.17$ & $96 \cdot 1$ & -0.25 & $-0.54,0.04$ \\
\hline \multicolumn{13}{|l|}{ Women $(n 816)$} \\
\hline$<23.5$ (lowest) & $123 \cdot 0$ & 0.00 & Referent & $76 \cdot 6$ & 0.00 & Referent & $46 \cdot 4$ & 0.00 & Referent & $92 \cdot 1$ & 0.00 & Referent \\
\hline $23 \cdot 5-31 \cdot 3$ & $122 \cdot 8$ & -0.01 & $-3.53,3.47$ & 77.4 & 0.94 & $-0.96,2.84$ & $45 \cdot 4$ & -0.97 & $-3.39,1.44$ & $92 \cdot 6$ & 0.62 & $-1.66,2.90$ \\
\hline $31 \cdot 4-38.7$ & $121 \cdot 6$ & -1.63 & $-5.31,2.05$ & $77 \cdot 2$ & 0.42 & $-1.58,2.42$ & 44.4 & -2.05 & $-4.59,0.49$ & $92 \cdot 0$ & -0.26 & $-2 \cdot 66,2 \cdot 13$ \\
\hline $38 \cdot 8-50.5$ & $125 \cdot 0$ & 1.98 & $-1 \cdot 74,5 \cdot 70$ & $79 \cdot 7$ & 2.92 & $0.90,4.94$ & $45 \cdot 4$ & -0.94 & $-3.50,1.63$ & 94.8 & $2 \cdot 61$ & $0.19,5.03$ \\
\hline$\geq 50.6$ (highest) & 121.9 & -0.90 & $-4.92,3.11$ & $77 \cdot 9$ & 1.28 & $-0.90,3.46$ & 43.9 & $-2 \cdot 19$ & $-4.96,0.57$ & $92 \cdot 6$ & 0.56 & $-2.05,3.17$ \\
\hline$P$ trend & & 0.94 & & & 0.06 & & & 0.16 & & & 0.27 & \\
\hline Continuous variable\| & 122.9 & -0.08 & $-2 \cdot 74,2 \cdot 59$ & $77 \cdot 7$ & 1.35 & $-0.08,2.79$ & $45 \cdot 2$ & -1.43 & $-3.28,0.43$ & $92 \cdot 8$ & 0.88 & $-0.85,2.60$ \\
\hline Each five unit increase & $122 \cdot 9$ & -0.02 & $-0.39,0.35$ & $77 \cdot 7$ & 0.18 & $-0.02,0.38$ & $45 \cdot 2$ & -0.20 & $-0.46,0.06$ & $92 \cdot 8$ & $0 \cdot 11$ & $-0.13,0.35$ \\
\hline \multicolumn{13}{|c|}{ Men and women combined ( $n$ 1076) } \\
\hline$<23.5$ (lowest) & 124.5 & 0.00 & Referent & 77.9 & 0.00 & Referent & $46 \cdot 7$ & 0.00 & Referent & 93.4 & 0.00 & Referent \\
\hline $23 \cdot 5-31 \cdot 3$ & $123 \cdot 8$ & -0.86 & $-4.05,2.32$ & $78 \cdot 3$ & 0.44 & $-1 \cdot 34,2 \cdot 22$ & $45 \cdot 4$ & -1.30 & $-3.50,0.90$ & 93.5 & 0.02 & $-2 \cdot 00,2 \cdot 11$ \\
\hline $31.4-38.7$ & 123.4 & -1.76 & $-5.04,1.52$ & $77 \cdot 8$ & -0.45 & $-2.29,1.39$ & $45 \cdot 6$ & -1.32 & $-3.58,0.95$ & 93.0 & -0.89 & $-3.05,1.28$ \\
\hline $38.8-50.5$ & $125 \cdot 5$ & 0.69 & $-2.62,4.01$ & $80 \cdot 2$ & 2.03 & $0.17,3.89$ & $45 \cdot 3$ & -1.33 & $-3.63,-0.96$ & $95 \cdot 3$ & 1.58 & $-0.61,3.77$ \\
\hline$\geq 50 \cdot 6$ (highest) & $122 \cdot 6$ & -2.42 & $-5.90,1.06$ & $77 \cdot 9$ & -0.11 & $-2 \cdot 06,1 \cdot 84$ & $44 \cdot 7$ & $-2 \cdot 26$ & $-4.67,-0.12$ & $92 \cdot 8$ & -0.88 & $-3 \cdot 18,1.42$ \\
\hline$P$ trend & & 0.42 & & & 0.53 & & & 0.09 & & & 0.96 & \\
\hline Continuous variable\| & $124 \cdot 0$ & -1.11 & $-3.62,1.41$ & 78.4 & 0.15 & $-1.26,1.57$ & $45 \cdot 5$ & -1.26 & $-3.00,0.48$ & 93.6 & -0.27 & $-1.93,1.40$ \\
\hline Each five unit increase & $124 \cdot 0$ & -0.13 & $-0.47,0.21$ & $78 \cdot 4$ & 0.02 & $-0.17,0.21$ & $45 \cdot 5$ & -0.15 & $-0.39,0.08$ & $93 \cdot 6$ & -0.03 & $-0.25,0.20$ \\
\hline
\end{tabular}

PP, pulse pressure; MAP, mean arterial pressure.

Note: Menopausal status and age were highly correlated $(r 0.81, P<0.01)$; so the two variables were not included in the same model for women. $\dagger \mathrm{PP}=$ systolic $\mathrm{BP}$ - diastolic $\mathrm{BP}$

$\S$ Derived from linear regression models adjusting for age (continuous), education (categorical), occupation, BMI (continuous), alcohol use (yes/no), smoking (yes/no), regular physical activity (yes/no), total physical activity (metabolic equivalents, $h /$ week; continuous), family history of hypertension (yes/no/unknown), intakes of total energy, eggs and vitamin $\mathrm{D}$ (continuous) and season of blood draw.

Log-transtormed 25(OH)D. 
Table 3. OR $(95 \% \mathrm{Cl})^{*}$ for the association between clinical categories of blood pressure (BP) and circulating 25-hydroxyvitamin D (25(OH)D) by sex in the Shanghai Women's Health Study and Shanghai Men's Health Study†

(Odd ratios and $95 \%$ confidence intervals)

\begin{tabular}{|c|c|c|c|c|c|c|c|c|c|c|c|c|}
\hline \multirow[b]{3}{*}{ 25(OH)D (nmol/l) } & \multicolumn{4}{|c|}{ Men ( $n$ 405) } & \multicolumn{4}{|c|}{ Women ( $n$ 1055) } & \multicolumn{4}{|c|}{ Combined $(n$ 1460)‡ } \\
\hline & \multicolumn{2}{|c|}{ Pre-hypertension } & \multicolumn{2}{|c|}{ Hypertension } & \multicolumn{2}{|c|}{ Pre-hypertension } & \multicolumn{2}{|c|}{ Hypertension } & \multicolumn{2}{|c|}{ Pre-hypertension } & \multicolumn{2}{|c|}{ Hypertension } \\
\hline & OR & $95 \% \mathrm{Cl}$ & OR & $95 \% \mathrm{Cl}$ & OR & $95 \% \mathrm{Cl}$ & OR & $95 \% \mathrm{Cl}$ & OR & $95 \% \mathrm{Cl}$ & OR & $95 \% \mathrm{Cl}$ \\
\hline$n$ & \multicolumn{2}{|c|}{155} & \multicolumn{2}{|c|}{193} & \multicolumn{2}{|c|}{452} & \multicolumn{2}{|c|}{354} & \multicolumn{2}{|c|}{607} & \multicolumn{2}{|c|}{547} \\
\hline$\%$ & \multicolumn{2}{|c|}{$38 \cdot 3$} & \multicolumn{2}{|c|}{47.6} & \multicolumn{2}{|c|}{$42 \cdot 8$} & \multicolumn{2}{|c|}{$33 \cdot 6$} & \multicolumn{2}{|c|}{$41 \cdot 6$} & \multirow{2}{*}{\multicolumn{2}{|c|}{$37 \cdot 5$}} \\
\hline \multicolumn{12}{|l|}{ NHANES/USA criteria } & \\
\hline Deficient $(<37.5)$ & 1.00 & Referent & 1.00 & Referent & 1.00 & Referent & 1.00 & Referent & 1.00 & Referent & 1.00 & Referent \\
\hline Insufficient $(37.5-74.9)$ & 0.57 & $0.26,1.22$ & 0.56 & $0.26,1 \cdot 21$ & $1 \cdot 11$ & $0.77,1.60$ & 1.09 & $0.73,1.63$ & 1.00 & $0.73,1.37$ & 1.00 & $0.71,1.40$ \\
\hline Sufficient $(\geq 75.0)$ & 0.46 & $0.14,1.56$ & 0.42 & $0.12,1.43$ & 1.51 & $0.49,4.60$ & 1.07 & $0.31,3.72$ & 0.94 & $0.44,2.03$ & 0.86 & $0.38,1.95$ \\
\hline \multirow{2}{*}{\multicolumn{13}{|c|}{ WHO criteria§ }} \\
\hline & & & & & & & & & & & & \\
\hline Deficient $(<27.5)$ & 1.00 & Referent & 1.00 & Referent & 1.00 & Referent & 1.00 & Referent & 1.00 & Referent & 1.00 & Referent \\
\hline \multirow{2}{*}{\multicolumn{13}{|c|}{ By quintile }} \\
\hline & & & & & & & & & & & & \\
\hline$<23.5$ (lowest) & 1.00 & Referent & 1.00 & Referent & 1.00 & Referent & 1.00 & Referent & 1.00 & Referent & 1.00 & Referent \\
\hline $23.5-31.3$ & 0.54 & $0.12,2.39$ & 0.25 & $0.06,1.08$ & 1.08 & $0.66,1.76$ & 0.95 & $0.55,1.63$ & 1.04 & $0.66,1.63$ & 0.82 & $0.51,1.33$ \\
\hline $31.4-38.7$ & 0.71 & $0.15,3.31$ & 0.35 & $0.08,1.59$ & 1.24 & $0.75,2.05$ & 0.91 & $0.52,1.60$ & 1.25 & $0.79,1.99$ & 0.93 & $0.57,1.53$ \\
\hline $38 \cdot 8-50.5$ & 0.50 & $0 \cdot 12,2 \cdot 13$ & 0.30 & $0.07,1.25$ & 1.41 & $0.83,2.41$ & 1.14 & $0.63,2.06$ & 1.29 & $0.80,2.08$ & 1.07 & $0.64,1.77$ \\
\hline$\geq 50.6$ (highest) & 0.33 & $0.08,1.35$ & 0.16 & $0.04,0.65$ & 1.06 & $0.61,1.85$ & 0.97 & $0.53,1.79$ & 0.91 & $0.56,1.48$ & 0.77 & $0.46,1.29$ \\
\hline$P$ trend & 0.09 & & 0.02 & & 0.48 & & 0.86 & & 0.92 & & 0.65 & \\
\hline Continuous variable\| & 0.54 & $0.25,1.21$ & 0.41 & $0.18,0.92$ & $1 \cdot 16$ & $0.77,1.74$ & 1.14 & $0.73,1.80$ & 1.00 & $0.71,1.42$ & 0.95 & $0.66,1.38$ \\
\hline Each five unit increase & 0.95 & $0.88,1.03$ & 0.94 & $0.88,1.02$ & 1.02 & $0.97,1.08$ & 1.02 & $0.96,1.09$ & 1.00 & $0.95,1.05$ & 1.00 & $0.95,1.05$ \\
\hline
\end{tabular}

NHANES, National Health and Nutrition Examination Survey

*Derived from polytomous logistic regression models adjusting for age (continuous), education (categorical), occupation, BMI continuous), alcohol use (yes/no), smoking (yes/no), regular physical activity (yes/no), total physical activity (metabolic equivalents, h/week; continuous), family history of hypertension (yes/no), intakes of total energy, eggs and vitamin D (all continuous) and season of blood draw.

$\dagger$ Note: Subjects with normal BP $(<120 / 80)$ and non-users of antihypertensive drugs) were the referent for both pre-hypertension (120-139/80-89 and non-users of antihypertensive drugs) and hypertension ( $\geq 140 / 90$ and/or users of antihypertensive drugs) categories.

‡ Additionally adjusted for sex.

$\S$ Guidelines on Food Fortification with Micronutrients, WHO/FAO.

|| Log-transformed 25(OH)D. 
was found between hypertension and non-deficient vitamin D status $(25(\mathrm{OH}) \mathrm{D} \geq 27.5 \mathrm{nmol} / \mathrm{l}$, WHO criteria) among men $(\mathrm{OR}=0 \cdot 29 ; 95 \%$ CI $0 \cdot 10,0 \cdot 82)$. The highest quintile of $25(\mathrm{OH}) \mathrm{D}(\mathrm{OR}=0 \cdot 16 ; 95 \%$ CI $0.04,0.65$ for $\geq 50.6 \mathrm{nmol} / \mathrm{l}$; $\left.P_{\text {trend }}=0.02\right)$, as well as continuous levels of $25(\mathrm{OH}) \mathrm{D}$ $(\mathrm{OR}=0.41 ; 95 \% \mathrm{CI} 0 \cdot 18,0 \cdot 92)$ and each $5 \mathrm{nmol} / 1$ increase in $25(\mathrm{OH}) \mathrm{D}(\mathrm{OR}=0.94 ; 95 \% \mathrm{CI} 0.88,1.02)$, were consistently and inversely associated with hypertension among men. Among women, no significant associations were observed between vitamin D status or circulating 25(OH)D level and pre-hypertension or hypertension. Interactions between sex and 25(OH)D level or vitamin D status on pre-hypertension and hypertension were statistically significant $\left(P_{\text {interaction }}<0.05\right.$ for all, data not shown).

\section{Discussion}

In this population-based study, we found a high prevalence of vitamin D deficiency $(55.8 \%$ using the NHANES-III, USA criteria; $29.9 \%$ using the WHO criteria) among middle-aged and elderly Chinese men and women in Shanghai. This rate is comparable to the rate found among Chinese people of the same age range in Beijing ${ }^{(27)}$. Overall, we found that higher levels of circulating $25(\mathrm{OH}) \mathrm{D}$ were not associated with BP measures. However, in sex-specific analyses, we observed significant inverse associations between 25(OH)D level and BP parameters including systolic BP, diastolic BP and MAP among men. Consistently, vitamin D non-deficiency or higher quintiles of $25(\mathrm{OH}) \mathrm{D}$ level were inversely associated with hypertension among men. There was no strong evidence for associations between circulating 25(OH)D levels and BP parameters and hypertension among women.

A possible mechanism through which vitamin D affects BP levels may be the involvement of calcitriol or 1,25 dihydroxy vitamin $\mathrm{D}(1,25(\mathrm{OH}) 2 \mathrm{D})$ in the renin-angiotensin system ${ }^{(2,28)}$. Tomaschitz et al. ${ }^{(28)}$ found inverse associations between circulating $25(\mathrm{OH}) \mathrm{D}$ and $1,25(\mathrm{OH}) 2 \mathrm{D}$ and circulating renin and angiotensin levels. It has been suggested that $1,25(\mathrm{OH}) 2 \mathrm{D}$ suppresses renin gene transcription by activating the vitamin D receptor, which binds the cyclic AMP-response element binding protein and blocks the renin gene promoter activity, thereby resulting in a decrease in renin production ${ }^{(2)}$. An elevated renin level was found to be related to increased $\mathrm{BP}$ via increasing angiotensin II, which causes arterial constriction and increased extracellular fluid volume ${ }^{(4)}$. A positive association between hypertension and vitamin D deficiency ${ }^{(29)}$, particularly among those with higher parathyroid hormone (PTH) level, has been previously reported ${ }^{(28)}$. Young et al. ${ }^{(30)}$ found that hypertensive men had a $36 \%$ higher serum PTH level and a slightly lower serum $1,25(\mathrm{OH}) 2 \mathrm{D}$ level than normotensive men. However, they did not observe this relationship in women. Thus, the modifying effect of sex on vitamin D-related changes in BP parameters and hypertension in our study may be explained either by the hypothesis that oestrogen regulates vitamin D activity through the autocrine/paracrine system or due to the sex-dependent response of PTH to vitamin D regulation ${ }^{(1,31)}$.
In general, the results of the present study are supported by previous epidemiological findings that suggest a beneficial effect related to sufficient levels of circulating vitamin D on elevated individual BP parameters and hypertension in the US population ${ }^{(9,10)}$. In the NHANES-III (1988-94) and NHANES 2001-06 cross-sectional studies, an inverse association between serum 25(OH)D levels and systolic BP was observed among non-Hispanic Blacks and Mexican Americans with no history of hypertension ${ }^{(10,15)}$. In the recent Insulin Resistance Atherosclerosis Family Study, higher 25(OH)D levels were associated with a decrease in both systolic and diastolic BP among Hispanic and African American populations ${ }^{(32)}$. In a study of a young Lebanese population (mean age 23.9 (sD 2.9) years), serum 25(OH)D was inversely associated with systolic BP in men, but not in women, which is similar to the findings of the present study. It has been suggested that vitamin D and oestrogen levels influence each other and play a role in maintaining Ca homeostasis ${ }^{(33)}$ Correction of oestrogen deficiency in postmenopausal women has been associated with increased $1,25(\mathrm{OH}) 2 \mathrm{D}$ production and $\mathrm{Ca}$ absorption ${ }^{(34)}$.

A recent review and meta-analysis of observational and intervention studies found a significant association between incident hypertension and lower $25(\mathrm{OH}) \mathrm{D}$ level in the metaanalysis of three cohorts ${ }^{(12)}$. In contrast, a study in Spain found a significant positive correlation between serum $25(\mathrm{OH}) \mathrm{D}$ levels and systolic and diastolic BP in Caucasian men (mean age 36 years). In men, this relationship was present only among those with the rare homozygous genotype of the SNP rs 154410 (also known as the Bsm1 polymorphism) in the vitamin $\mathrm{D}$ receptor gene ${ }^{(8)}$. A similar tendency was found for PP in their follow-up study of Caucasian men with the BsmI genotype. No such association was found in women ${ }^{(35)}$. These studies suggest that the combined effect of BsmI vitamin D receptor gene polymorphisms and circulating $25(\mathrm{OH}) \mathrm{D}$ on $\mathrm{BP}$ may be altered by oestrogen ${ }^{(8,35)}$. In addition, Argiles et al. ${ }^{(16)}$ observed a positive association between systolic and diastolic BP and seasonal variations of circulating $25(\mathrm{OH}) \mathrm{D}$ among haemodialysis patients (mean age: 56 years). However, the Longitudinal Aging Study Amsterdam, which included 1205 older Caucasian men and women $(\geq 65 \text { years })^{(13)}$, and a small population-based, cross-sectional study of men ${ }^{(36)}$ found that neither systolic BP nor diastolic BP was associated with circulating $25(\mathrm{OH}) \mathrm{D}$ level. Only two studies have previously evaluated the association between plasma $25(\mathrm{OH}) \mathrm{D}$ and $\mathrm{BP}$ in a Chinese population and found contradicting results ${ }^{(27,37)}$. Lu et al. ${ }^{(27)}$ found an inverse association between plasma $25(\mathrm{OH}) \mathrm{D}$ and diastolic BP in the combined analyses of 1443 men and 1819 women aged 50-70 years from Beijing and Shanghai in China. In contrast, Chan et al. ${ }^{(37)}$ found no association between serum 25(OH)D and systolic BP or diastolic BP among elderly men in Hong Kong. This inconsistent evidence may be due to differences in vitamin D status in these two study populations ${ }^{(37)}$. More studies are needed to evaluate the sex influence of the association between circulating vitamin D levels and BP.

Many intervention studies have examined the effect of vitamin D supplementation on BP parameters and provided 
inconsistent results ${ }^{(12)}$, and few have evaluated the effect in relation to circulating $25(\mathrm{OH}) \mathrm{D}$ levels. A double-blind, placebo-controlled study in Sweden found an inverse association between $25(\mathrm{OH}) \mathrm{D}$ and both systolic and diastolic BP, with the use of vitamin D supplements (cholecalciferol or vitamin $\mathrm{D}_{3}$ ), among middle-aged individuals with normal BP and normal weight. This study also found a decrease in BP with vitamin D supplement use among patients with mild and moderate hypertension, particularly among those with primary hyperparathyroidism or low plasma renin activity ${ }^{(38,39)}$. Similarly, another intervention study in Germany found a decrease in systolic BP after 8 weeks of vitamin D and Ca supplementation in elderly women with insufficient levels of $25(\mathrm{OH}) \mathrm{D}$ $(<50.0 \mathrm{nmol} / 1)^{(40)}$. In contrast, in a recent meta-analysis of ten randomised trials, vitamin D supplementation was not significantly associated with either systolic or diastolic $\mathrm{BP}^{(12)}$

Longitudinal observational studies have also investigated the relationship between circulating $25(\mathrm{OH}) \mathrm{D}$ and hypertension, and most of these studies have found an inverse association between hypertension and $25(\mathrm{OH}) \mathrm{D}$ level in both women and men of European ancestry ${ }^{(9,12)}$. In the Health Professionals' Study and Nurses' Health Study in the USA, an increased risk of incident hypertension was independently associated with lower 25(OH)D levels in both men and women $^{(9)}$. Similarly, in NHANES-III, lower serum 25(OH)D levels were significantly associated with CVD risk factors, including hypertension, among the elderly (aged $\geq 60$ years) non-Hispanic African and Mexican American populations ${ }^{(41)}$. However, the Longitudinal Aging Study Amsterdam study found no association between the risk of hypertension and vitamin D status among elderly individuals ${ }^{(13)}$. Several other studies found no effect of circulating $25(\mathrm{OH}) \mathrm{D}$ on the metabolic syndrome, including hypertension in both women and men ${ }^{(42,43)}$. In contrast, a recent systematic review and metaanalysis of twenty-eight studies including 99745 participants found a $43 \%$ reduction in cardiometabolic disorders, including CVD, diabetes and the metabolic syndrome, associated with the highest levels of circulating $25(\mathrm{OH}) \mathrm{D}$ in a middleaged and elderly population ${ }^{(44)}$.

In intervention studies on the use of UV light, both UVB and UVA have been shown to decrease BP. One study observed a significant decrease in systolic BP and normalised $25(\mathrm{OH}) \mathrm{D}$ level with the use of full-body UVB light (three times weekly for $8 \mathrm{~min}$, which is equivalent to a 12 - to $24-\mathrm{min}$ exposure to sunlight) in women (aged 26-66 years) with untreated mild hypertension and low plasma 25(OH)D) levels $(<50 \cdot 0 \mathrm{nmol} / 1)^{(45)}$. Another study found that systolic BP significantly decreased with the use of UVB/UVA lights in both Caucasian and African American women without or with hypertension ${ }^{(46)}$. Similarly, a recent experimental study of healthy volunteers showed that BP parameters (systolic $\mathrm{BP}$, diastolic BP and MAP) were significantly lower after exposure to UVA light for up to $60 \mathrm{~min}^{(47)}$. Although the results from these intervention studies are not directly comparable to our findings, they clearly provided additional support for the association between the levels of BP parameters and circulating 25(OH)D.
Our study has several limitations. The association between vitamin D status and risk of high $\mathrm{BP}$ could have been obscured by the effect of seasonal variation on the blood samples and demographic, dietary and lifestyle factors, and medical history. However, we carefully adjusted for many of these factors in the statistical analyses. Nevertheless, the possibility of residual confounding remains. The relatively small number of individuals with sufficient levels of vitamin $\mathrm{D}$ ( $\geq 75 \mathrm{nmol} / 125(\mathrm{OH}) \mathrm{D}$ ) in this study prevented us from conducting any subgroup analyses. Another limitation is the use of only a single measurement of $25(\mathrm{OH}) \mathrm{D}$, which may have led to an underestimation of the association between vitamin D status and BP levels. However, prior studies have shown that circulating $25(\mathrm{OH}) \mathrm{D}$ concentration is relatively stable over time ${ }^{(7)}$. Our study is also limited because the clinical categories of BP (normotensive, pre-hypertensive or hypertensive) were classified by using BP measurements taken in a single home visit and self-reported antihypertensive medication use, which could have led to misclassification of disease status. However, misclassification in the assessment of hypertension is likely to be independent of $25(\mathrm{OH}) \mathrm{D}$ level. Thus, the misclassifications are most likely to be non-differential and result in an underestimation of the true association. Lastly, we did not collect information on plasma/serum PTH; we were therefore unable to investigate possible confounding or modifying effects on the associations of $25(\mathrm{OH}) \mathrm{D}$ levels with BP.

This study has several notable strengths, including the use of data from population-based cohort studies with high response rates and a relatively large sample size, which minimised selection bias, and the uniqueness of the geographic location, lifestyle, dietary characteristics and biological samples represented by our participants. Circulating $25(\mathrm{OH}) \mathrm{D}$ level was measured using blood samples that were collected before or during the BP measurement. BP was measured by retired medical professionals at the participants' home according to a standard protocol. Unlike foods in many Western countries, the Chinese food supply is not fortified with vitamin D. Therefore, plasma $25(\mathrm{OH}) \mathrm{D}$ concentrations tend to be consistent. This enabled us to investigate the effect of vitamin D status, which was mainly determined by natural exposures, on $\mathrm{BP}$

In summary, we found that circulating $25(\mathrm{OH}) \mathrm{D}$ levels are inversely associated with levels of individual BP parameters and the prevalence of hypertension among middleaged and elderly Chinese men but not among women. Further studies are needed to confirm this sex-specific association and to identify the underlying mechanisms.

\section{Acknowledgements}

The contributions of the authors were as follows: T. D. analysed the data and drafted the manuscript. X. O. S. and W. Z. designed and directed the study, obtained funding for the parent studies and provided critical review of the manuscript. Y.-B. X., G. Y., H. L. and Y.-T. G. directed and supervised the field operations of the parent studies, data cleaning and reviewed the manuscript. B.-T. J., G. Y. and Q. C. contributed to critical review of the paper. H. C. contributed to the 
statistical analysis and reviewed the paper. The authors thank the Shanghai residents who participated in the studies and the research staff of the Shanghai Women's Health Study (SWHS) and the Shanghai Men's Health Study (SMHS) for their dedication and contributions to the present study. The authors also thank Bethanie Rammer and Jacqueline Stern for their editorial assistance. This work was supported by the National Cancer Institute at the National Institutes of Health, USA (R37 CA070867, R01 CA082729, R01 HL079123). The authors declare that they have no conflicts of interest.

\section{References}

1. Kinuta K, Tanaka H, Moriwake T, et al. (2000) Vitamin D is an important factor in estrogen biosynthesis of both female and male gonads. Endocrinology 141, 1317-1324.

2. Li YC, Qiao G, Uskokovic M, et al. (2004) Vitamin D: a negative endocrine regulator of the renin-angiotensin system and blood pressure. J Steroid Biochem Mol Biol 89-90, 387-392.

3. Holick MF (2005) Vitamin D: important for prevention of osteoporosis, cardiovascular heart disease, type 1 diabetes, autoimmune diseases, and some cancers. South Med J 98, 1024-1027.

4. Martini LA \& Wood RJ (2008) Vitamin D and blood pressure connection: update on epidemiologic, clinical, and mechanistic evidence. Nutr Rev 66, 291-297.

5. O'Donnell S, Cranney A, Horsley T, et al. (2008) Efficacy of food fortification on serum 25-hydroxyvitamin D concentrations: systematic review. Am J Clin Nutr 88, 1528-1534.

6. Holden JM \& Lemar LE (2008) Assessing vitamin D contents in foods and supplements: challenges and needs. Am J Clin Nutr 88, 551S-553S.

7. Holick MF (2009) Vitamin D status: measurement, interpretation, and clinical application. Ann Epidemiol 19, 73-78.

8. Muray S, Parisi E, Cardus A, et al. (2003) Influence of vitamin $\mathrm{D}$ receptor gene polymorphisms and 25-hydroxyvitamin D on blood pressure in apparently healthy subjects. J Hypertens 21, 2069-2075.

9. Forman JP, Giovannucci E, Holmes MD, et al. (2007) Plasma 25-hydroxyvitamin D levels and risk of incident hypertension. Hypertension 49, 1063-1069.

10. Scragg R, Sowers M \& Bell C (2007) Serum 25-hydroxyvitamin $\mathrm{D}$, ethnicity, and blood pressure in the Third National Health and Nutrition Examination Survey. Am J Hypertens 20, 713-719.

11. Scragg RK, Camargo CA Jr \& Simpson RU (2010) Relation of serum 25-hydroxyvitamin D to heart rate and cardiac work (from the National Health and Nutrition Examination Surveys). Am J Cardiol 105, 122-128.

12. Pittas AG, Chung M, Trikalinos T, et al. (2010) Systematic review: vitamin D and cardiometabolic outcomes. Ann Intern Med 152, 307-314.

13. Snijder MB, Lips P, Seidell JC, et al. (2007) Vitamin D status and parathyroid hormone levels in relation to blood pressure: a population-based study in older men and women. J Intern Med 261, 558-565.

14. Nagpal J, Pande JN \& Bhartia A (2009) A double-blind, randomized, placebo-controlled trial of the short-term effect of vitamin $D_{3}$ supplementation on insulin sensitivity in apparently healthy, middle-aged, centrally obese men. Diabet Med 26, 19-27.

15. Scragg R, Khaw KT \& Murphy S (1995) Effect of winter oral vitamin $\mathrm{D}_{3}$ supplementation on cardiovascular risk factors in elderly adults. Eur J Clin Nutr 49, 640-646.
16. Argiles A, Lorho R, Servel MF, et al. (2002) Blood pressure is correlated with vitamin $\mathrm{D}(3)$ serum levels in dialysis patients. Blood Purif 20, 370-375.

17. Dorjgochoo T, Shu XO, Zhang X, et al. (2009) Relation of blood pressure components and categories and all-cause, stroke and coronary heart disease mortality in urban Chinese women: a population-based prospective study. J Hypertens 27, 468-475.

18. Fang XH, Longstreth WT Jr, Li SC, et al. (2001) Longitudinal study of blood pressure and stroke in over 37,000 People in China. Cerebrovasc Dis 11, 225-229.

19. Jurj AL, Wen W, Xiang YB, et al. (2007) Reproducibility and validity of the Shanghai Men's Health Study physical activity questionnaire. Am J Epidemiol 165, 1124-1133.

20. Zheng W, Chow WH, Yang G, et al. (2005) The Shanghai Women's Health Study: rationale, study design, and baseline characteristics. Am J Epidemiol 162, 1123-1131.

21. Gallicchio L, Helzlsouer KJ, Chow WH, et al. (2010) Circulating 25-hydroxyvitamin $\mathrm{D}$ and the risk of rarer cancers: design and methods of the cohort consortium vitamin D pooling project of rarer cancers. Am J Epidemiol 172, 10-20.

22. Chobanian AV, Bakris GL, Black HR, et al. (2003) Seventh report of the Joint National Committee on Prevention, Detection, Evaluation, and Treatment of High Blood Pressure. Hypertension 42, 1206-1252.

23. Shu XO, Yang G, Jin F, et al. (2004) Validity and reproducibility of the food frequency questionnaire used in the Shanghai Women's Health Study. Eur J Clin Nutr 58, 17-23.

24. Yang G, Wang G \& Pan X (2002) China Food Composition. Beijing: Peking University Medical Press.

25. Hollis BW (2005) Circulating 25-hydroxyvitamin D levels indicative of vitamin D sufficiency: implications for establishing a new effective dietary intake recommendation for vitamin D. J Nutr 135, 317-322.

26. World Health Organization and Food and Agricultural Organization of the United Nations (2004) Vitamin and Mineral Requirements in Human Nutrition. Geneva: WHO.

27. Lu L, Yu Z, Pan A, et al. (2009) Plasma 25-hydroxyvitamin D concentration and metabolic syndrome among middleaged and elderly Chinese individuals. Diabetes Care $\mathbf{3 2}$ $1278-1283$.

28. Tomaschitz A, Pilz S, Ritz E, et al. (2010) Independent association between 1,25-dihydroxyvitamin D, 25-hydroxyvitamin $\mathrm{D}$ and the renin-angiotensin system: the Ludwigshafen Risk and Cardiovascular Health (LURIC) study. Clin Chim Acta 411, 1354-1360.

29. Wang TJ, Pencina MJ, Booth SL, et al. (2008) Vitamin D deficiency and risk of cardiovascular disease. Circulation 117, 503-511.

30. Young EW, McCarron DA \& Morris CD (1990) Calcium regulating hormones in essential hypertension. Importance of gender. Am J Hypertens 3, 161S-166S.

31. Gunnarsson O, Indridason OS, Franzson L, et al. (2009) Factors associated with elevated or blunted PTH response in vitamin D insufficient adults. J Intern Med 265, 488-495.

32. Schmitz KJ, Skinner HG, Bautista LE, et al. (2009) Association of 25-hydroxyvitamin D with blood pressure in predominantly 25-hydroxyvitamin D deficient Hispanic and African Americans. Am J Hypertens 22, 867-870.

33. Gannage-Yared MH, Chedid R, Khalife S, et al. (2009) Vitamin D in relation to metabolic risk factors, insulin sensitivity and adiponectin in a young Middle-Eastern population. Eur J Endocrinol 160, 965-971.

34. Gallagher JC \& Sai AJ (2010) Vitamin D insufficiency, deficiency, and bone health. J Clin Endocrinol Metab 95 $2630-2633$. 
35. Muray S, Marco MP, Craver L, et al. (2006) Influence of mineral metabolism parameters on pulse pressure in healthy subjects. Clin Nephrol 66, 411-417.

36. Reichel H, Liebethal R, Hense HW, et al. (1992) Disturbed calcium metabolism in subjects with elevated diastolic blood pressure. Clin Investig 70, 748-751.

37. Chan R, Chan D, Woo J, et al. (2011) Serum 25-hydroxyvitamin $\mathrm{D}$ and parathyroid hormone levels in relation to blood pressure in a cross-sectional study in older Chinese men. J Hum Hypertens (epublication ahead of print version 20 January 2011).

38. Lind L, Wengle B, Wide L, et al. (1988) Hypertension in primary hyperparathyroidism-reduction of blood pressure by long-term treatment with vitamin $\mathrm{D}$ (alphacalcidol). A double-blind, placebo-controlled study. Am J Hypertens 1, 397-402.

39. Lind L, Wengle B, Wide L, et al. (1989) Reduction of blood pressure during long-term treatment with active vitamin $\mathrm{D}$ (alphacalcidol) is dependent on plasma renin activity and calcium status. A double-blind, placebo-controlled study. Am J Hypertens 2, 20-25.

40. Pfeifer M, Begerow B, Minne HW, et al. (2001) Effects of a short-term vitamin $\mathrm{D}(3)$ and calcium supplementation on blood pressure and parathyroid hormone levels in elderly women. J Clin Endocrinol Metab 86, 1633-1637.
41. Martins D, Wolf M, Pan D, et al. (2007) Prevalence of cardiovascular risk factors and the serum levels of 25-hydroxyvitamin D in the United States: data from the Third National Health and Nutrition Examination Survey. Arch Intern Med 167, 1159-1165.

42. Jorde R, Figenschau Y, Emaus N, et al. (2010) Serum 25-hydroxyvitamin D levels are strongly related to systolic blood pressure but do not predict future hypertension. Hypertension 55, 792-798.

43. Rueda S, Fernandez-Fernandez C, Romero F, et al. (2008) Vitamin D, PTH, and the metabolic syndrome in severely obese subjects. Obes Surg 18, 151-154.

44. Parker J, Hashmi O, Dutton D, et al. (2009) Levels of vitamin $\mathrm{D}$ and cardiometabolic disorders: systematic review and meta-analysis. Maturitas 65, 225-236.

45. Krause R, Buhring M, Hopfenmuller W, et al. (1998) Ultraviolet B and blood pressure. Lancet 352, 709-710.

46. Weber KT, Rosenberg EW \& Sayre RM (2004) Suberythemal ultraviolet exposure and reduction in blood pressure. Am J Med 117, 281-282.

47. Oplander C, Volkmar CM, Paunel-Gorgulu A, et al. (2009) Whole body UVA irradiation lowers systemic blood pressure by release of nitric oxide from intracutaneous photolabile nitric oxide derivates. Circ Res 105, 1031-1040. 